\title{
3D-QSAR analysis of pyrimidine derivatives as AXL kinase inhibitors as anticancer agents
}

\author{
Siddharth J. Modi, Vithal M. Kulkarni* \\ Department of Pharmaceutical Chemistry, Poona College of Pharmacy, Bharati Vidyapeeth (Deemed to be University), Pune- 411038, Maharashtra, India.
}

\section{ARTICLE INFO \\ Article history: \\ Received on: 06/06/2018 \\ Accepted on: 08/07/2018 \\ Available online: 30/11/2018}

\section{Key words:}

AXL kinase receptor,

Pyrimidine derivatives,

3D-QSAR, CoMFA,

COMSIA.

\begin{abstract}
AXL kinase receptor belongs to the TAM family of receptor tyrosine kinases (RTKs). Different types of cancer namely breast cancer, osteosarcoma, acute myeloid leukemia, colorectal cancer and non-small cell lung cancer (NSCLC) manifest overexpression of AXL receptor. Moreover, AXL kinase overexpression leads to tumor angiogenesis \& resistance to chemotherapeutic agents and reduces the antitumor immune response. Therefore, AXL kinase has emerged as the potential and attractive target for the treatment of cancer. The present study is based on the correlation between the structural parameter and biological activity of the compounds using the 3D-QSAR technique. In this technique, pyrimidine derivatives and their inhibitory activity against AXL kinase receptor were chosen as independent and dependent variables respectively. Based on the investigation, the structural requirements for AXL kinase inhibition were recognized. Here, CoMFA and CoMSIA analysis were used for the execution of the 3D-QSAR model. The training and the test set pyrimidine derivatives were used for the generation and validation of QSAR model respectively. Dataset alignment was performed using the lowest energy conformer of the most active compound. CoMFA, as well as CoMSIA, model have encouraging values of the cross-validation coefficient $\left(\mathrm{q}^{2}\right) 0.700$ and 0.622 and conventional correlation coefficient $\left(\mathrm{r}^{2}\right) 0.911$ and 0.875 independently. Furthermore, values of $\mathrm{r}^{2}$ pred were obtained as 0.709 and 0.668 respectively. Outcomes of the QSAR models and contour maps may be used for discovery of new AXL kinase inhibitors as potent anticancer agents.
\end{abstract}

\section{INTRODUCTION}

Receptor tyrosine kinases (RTKs) are the type of multidomain transmembrane proteins. These proteins are the sensor for extracellular ligands (O'Bryan et al., 1991). When ligands bind to the active site of the receptor, the receptor dimerization and activation of intracellular kinase domain were achieved. This biochemical process leads to the recruitment, phosphorylation, and activation of multiple downstream signaling cascades. The AXL tyrosine kinase receptor is a member of the TAM (TYRO3-AXLMER) group of RTKs and emerged as the promising therapeutic target for cancer therapy (Graham et al., 2014; Lai et al., 1994). Other than AXL kinase, TYRO-3 (also known as Brt, Dtk, Rse, Sky and Tif), and MER (also known as Eyk, Nym and Tyro12)

*Corresponding Author

Dr. Vithal M. Kulkarni, Professor Emeritus, Department of

Pharmaceutical Chemistry, Poona College of Pharmacy, Bharati

Vidyapeeth (Deemed to be University), Erandwane, Pune-411038,

Maharashtra, India.E-mail:vmkulkarni60@gmail.com receptors are also part of TAM receptor kinase. The overexpression of AXL has been reported in different types of cancer namely nonsmall cell lung cancer (NSCLC), osteosarcoma, breast cancer, acute myeloid leukemia, prostate and colorectal cancer (Zhang et al., 2013; Paccez et al., 2013; Ou et al., 2011; Yuen et al., 2013). Further, epithelial-mesenchymal transition (EMT) because of AXL signaling mechanism in tumor cells leads to the development of drug resistance to targeted therapies and chemotherapy (Hong et al., 2008; Zhang et al., 2012; Zhou et al., 2016). Most of AXL kinase inhibitors were discovered due to the similarity in the kinase domain of AXL receptor with MET or MER receptors (Gay et al., 2017). Bemcentinib (BGB324 or R428) is one of the most advance AXL selective inhibitor. It is an oral small molecule in the second phase of the clinical trial for NSCLC management (Ben-Batalla et al., 2013). Non-selective AXL kinase inhibitors include foretinib, carbozantinib, merestinib, amuvatinib, bosutinib, gilteritinib etc. (Eder et al., 2010; Hart et al., 2013; Lee et al., 2013; Yan et al., 2013; Mahadevan et al., 2007; Mori et al., 2014). The structures of AXL kinase receptor inhibitors are depicted in Figure 1. 
The selective and potent inhibitory activity of the series of novel pyrimidine derivatives towards AXL kinase has been reported (Mollard et al., 2011). The correlation of the structural parameter (independent variable) with the biological activity (dependent variable) of the derivatives was performed using the 3D-QSAR technique. Here, CoMFA and CoMSIA methods were accomplished for 3D-QSAR model development. Comparative molecular field analysis (CoMFA) is the type of 3D-QSAR technique where, steric and electrostatic fields were used as an independent variable against the biological activity of compounds (Cramer et al., 1988). Whereas, CoMSIA is improved method of CoMFA in which other than steric and electrostatic, hydrophobic, hydrogen bond donor and hydrogen bond acceptor were used as an independent variable for the correlation with the biological activity of the compounds (Klebe et al., 1994).

We have generated the quantitative structure-activity relationship (QSAR) model of pyrimidine derivatives for the prediction of biological activity. Based, on the QSAR model 3D contour maps of steric, electrostatic, hydrophobic, H-bond donor and $\mathrm{H}$-bond acceptor fields were generated and SAR was established. These details could be applied to modify the structure and improve the biological activity of pyrimidine derivatives against AXL kinase receptor.

\section{MATERIALS AND METHODS}

\section{Dataset preparation}

We have collected the pyrimidine derivatives as AXL kinase inhibitors for this study from the literature (Mollard et al., 2011). The pyrimidine derivatives were bifurcated into approximate $70 \%$ of the training set (18 derivatives) and approximate $30 \%$ of the test set ( 7 derivatives). Compounds in the test set have a range of biological activity similar to the training set. Derivatives present in the training set and test set were used for the generation and validation of QSAR model respectively. The $\mathrm{IC}_{50}$ value of all derivatives was transformed into $\mathrm{pIC}_{50}\left(-\operatorname{logIC} \mathrm{C}_{50}\right)$ value. These transformed $\mathrm{pIC}_{50}$ values were utilized as a dependent variable for CoMFA and CoMSIA model generation (Table 1) (Liu et al., 2011). Table 1 depicts the structure of all pyrimidine derivatives along with their biological activity against AXL kinase receptor.<smiles>Nc1nc(Nc2ccc3c(c2)CCC(N2CCCC2)CC3)nn1-c1cc2c(nn1)-c1ccccc1CCC2</smiles>

Bemcentinib<smiles>COc1cc2nccc(Oc3ccc(NC(=O)C4(C(=O)Nc5ccc(F)cc5)CC4)cc3)c2cc1OC</smiles>

Carbozantinib<smiles>COc1cc2c(Oc3ccc(NC(=O)C4(C(=O)Nc5ccc(F)cc5)CC4)cc3F)ccnc2cc1OCCCN1CCOCC1</smiles>

Foretinib

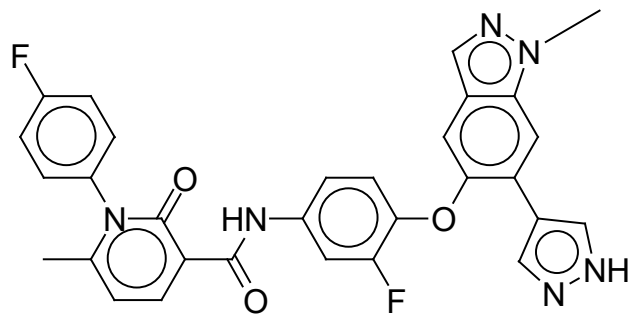

Merestinib

Fig. 1: Chemical structure of AXL tyrosine kinase inhibitors.

\section{Computational study and alignment of database}

SYBYL-X 2.0 from Tripos Inc. was used to perform the 3D-QSAR study (SYBYL X Molecular Modeling Software). In the SYBYL software, SKETCH function was utilized for construction of the 3D structure of all pyrimidine derivatives. Gasteiger Huckel (GH) charges along with the TRIPOS force field was applied to all designed derivatives for structure optimization process. Further, minimization was carried out by Powell conjugated gradient algorithm method (Clark et al., 1989). Structure alignment is the utmost important step in QSAR study. Here, Distill alignment function was carried out. Alignment was executed by selecting highly potent pyrimidine derivative from the dataset as template structure (Raichurkar et al., 2003). Figure 2 and Figure 3 represent a common fragment of AXL kinase inhibitors and all aligned compounds respectively.

\section{CoMFA and CoMSIA model}

Three-dimensional cubic lattice with $2 \AA$ grid space, which was extended to $4 \AA$ for the aligned dataset in $X, Y$, and $\mathrm{Z}$ three axis, was defined automatically to calculate the CoMFA and CoMSIA fields. Here, $\mathrm{Sp}^{3}$ carbon atom with the radius of 1.52 $\AA$ and +1 charge was used as probe atom under CoMFA analysis (Sridhar et al., 2011). In CoMFA analysis, for the derivation of steric and electrostatic fields, interaction energy was calculated using the Tripos force field. $\pm 30 \mathrm{kcal} / \mathrm{mol}$ cut-off value was used for the steric and electrostatic fields respectively. At the lattice 
intersection, Lennard-Jones and Columbic potentials were used (Bush et al., 1993).

In the CoMSIA, five descriptors namely steric, electrostatic, hydrophobic, H-bond donor and H-bond acceptor were used (Myint et al., 2010). For hydrophobic, H-bond donor and $\mathrm{H}$-bond acceptor, probe atom with +1 charge was used by default. While all other parameters are similar as in the CoMFA model, only the value of attenuation factor $(\alpha)$ was set to 0.3 for ease of functioning (Buolamwini et al., 2002).
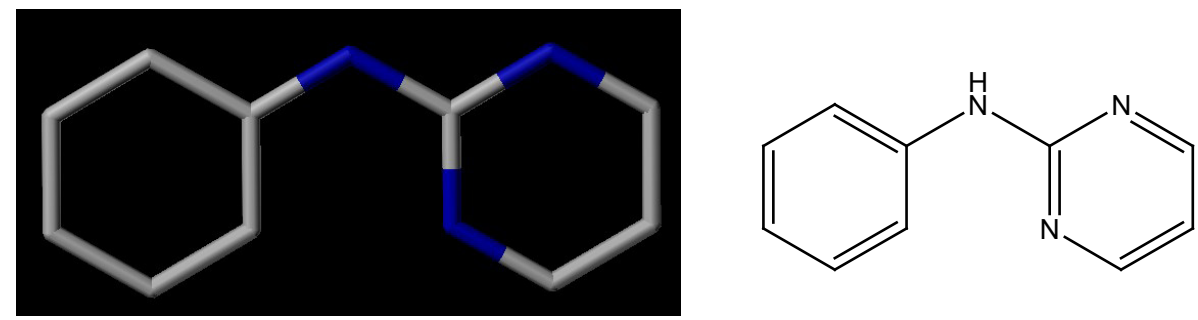

Fig. 2: The common fragment of AXL kinase inhibitors for alignment of the database to perform QSAR study.

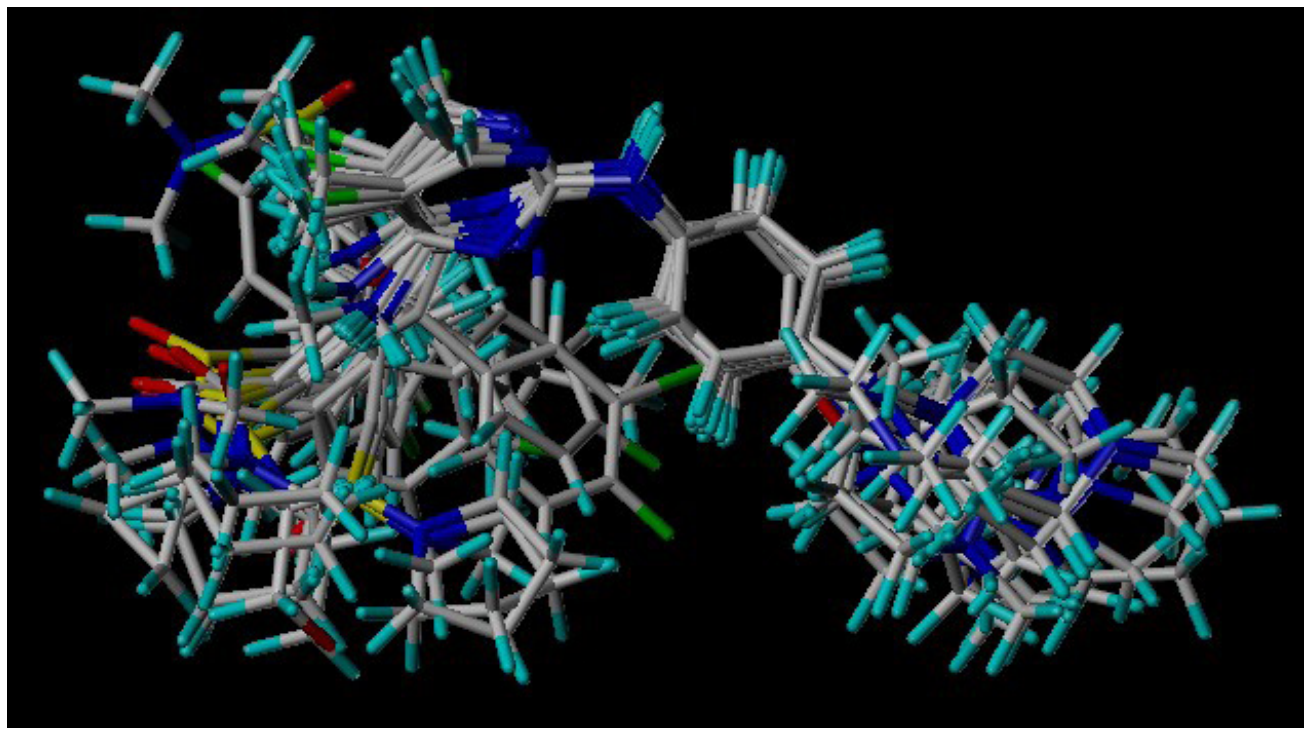

Fig. 3: Dataset alignment for 3D-QSAR (CoMFA and CoMSIA) study.

\section{PLS analysis}

PLS (Partial Least Square) analysis is useful when so many highly colinear factors are present. It is an extension of multiple linear regression analysis (MLR) method. The 3D-QSAR model was built using PLS analysis. Here, the values of CoMFA and CoMSIA were used as an independent variable, while AXL kinase inhibitory activity was used as a dependent variable for the correlation of the structural parameters with its biological activity. If the QSAR model is developed using the optimum number of components with a high value of cross-validated coefficient $\left(\mathrm{q}^{2}\right)$ and low standard errors, then chances of overfitted models are very less. In cross-validated coefficient $\left(\mathrm{q}^{2}\right)$ method, one compound was removed from the set and by use of all other compounds, the model was generated. The activity of the elided compound was predicted using the developed QSAR model. If methods give a $\mathrm{q}^{2}$ value $>0.5$ and $\mathrm{r}^{2}>0.616$, models are termed as acceptable (Golbraikh et al., 2002). It is estimated by the following equation:

$$
\mathrm{q}^{2}=1-\frac{\sum\left(\mathrm{Y}_{\text {predicted }}-\mathrm{Y}_{\text {observed }}\right)}{\sum\left(\mathrm{Y}_{\text {observed- }} \mathrm{Y}_{\text {mean }}\right)}
$$

The optimum number of component (ONC) was used to calculate the value of $\mathrm{r}_{\text {ncv }}^{2}$ (non-cross validated correlation coefficient). Further, the robustness of the developed model was checked by bootstrap analysis. In this method, from the entire dataset random compounds were selected. This method was undertaken many times (minimum 100 times for more accuracy). In each run, some compounds were elided from the dataset while some compounds were included in the dataset. The value of $\mathrm{r}_{\mathrm{bs}}^{2}$ from bootstrap analysis and $\mathrm{r}_{\mathrm{cv}}^{2}$ from cross-validation were determined (Lu et al., 2010).

\section{Predictive correlation coefficient $\left(\mathrm{r}^{2}{ }_{\text {pred }}\right)$}

The compounds, which were not incorporated in the training set (7 test set compounds), were used for the calculation of predictability of generated QSAR model. Following equation was used to calculate the value of $\mathrm{r}^{2}{ }_{\text {pred }}$ :

$$
r_{\text {pred }}^{2}=\frac{(S D-P R E S S)}{S D}
$$

where SD, is the sum of the squared deviations between the biological activity of the test set and mean activity of the training 
set compounds, and PRESS is the sum of squared deviations

in the test set (Makhija et al., 2002).

between actual and predicted activity values for each compound

Table 1: Structure of pyrimidine derivatives, experimental activity and predicted inhibitory activity of training set and test set compounds using 3D-QSAR (CoMFA and CoMSIA) method.

\begin{tabular}{|c|c|c|c|c|c|c|c|}
\hline \multirow{2}{*}{ Compound No. } & \multirow{2}{*}{ Compound } & \multirow{2}{*}{$\mathrm{IC}_{50}{ }^{i}(\mu \mathrm{M})$} & \multirow{2}{*}{$\mathbf{p I C}_{50}{ }^{i i}$} & \multicolumn{2}{|c|}{ CoMFA } & \multicolumn{2}{|c|}{ CoMSIA } \\
\hline & & & & Pred $^{\text {iii }}$ & $\operatorname{Res}^{\mathrm{iv}}$ & Pred $^{v}$ & Res $^{\text {vi }}$ \\
\hline $3 \#$ & & 2.80 & 5.552 & 5.623 & -0.071 & 5.627 & -0.075 \\
\hline $4 \#$ & & 6.10 & 5.214 & 5.067 & 0.147 & 5.250 & -0.036 \\
\hline 5 & & 0.73 & 6.136 & 6.233 & -0.097 & 6.005 & 0.131 \\
\hline 6 & & 1.63 & 5.787 & 5.857 & -0.07 & 5.973 & -0.186 \\
\hline 7 & & 0.42 & 6.376 & 6.407 & -0.031 & 6.589 & -0.213 \\
\hline
\end{tabular}




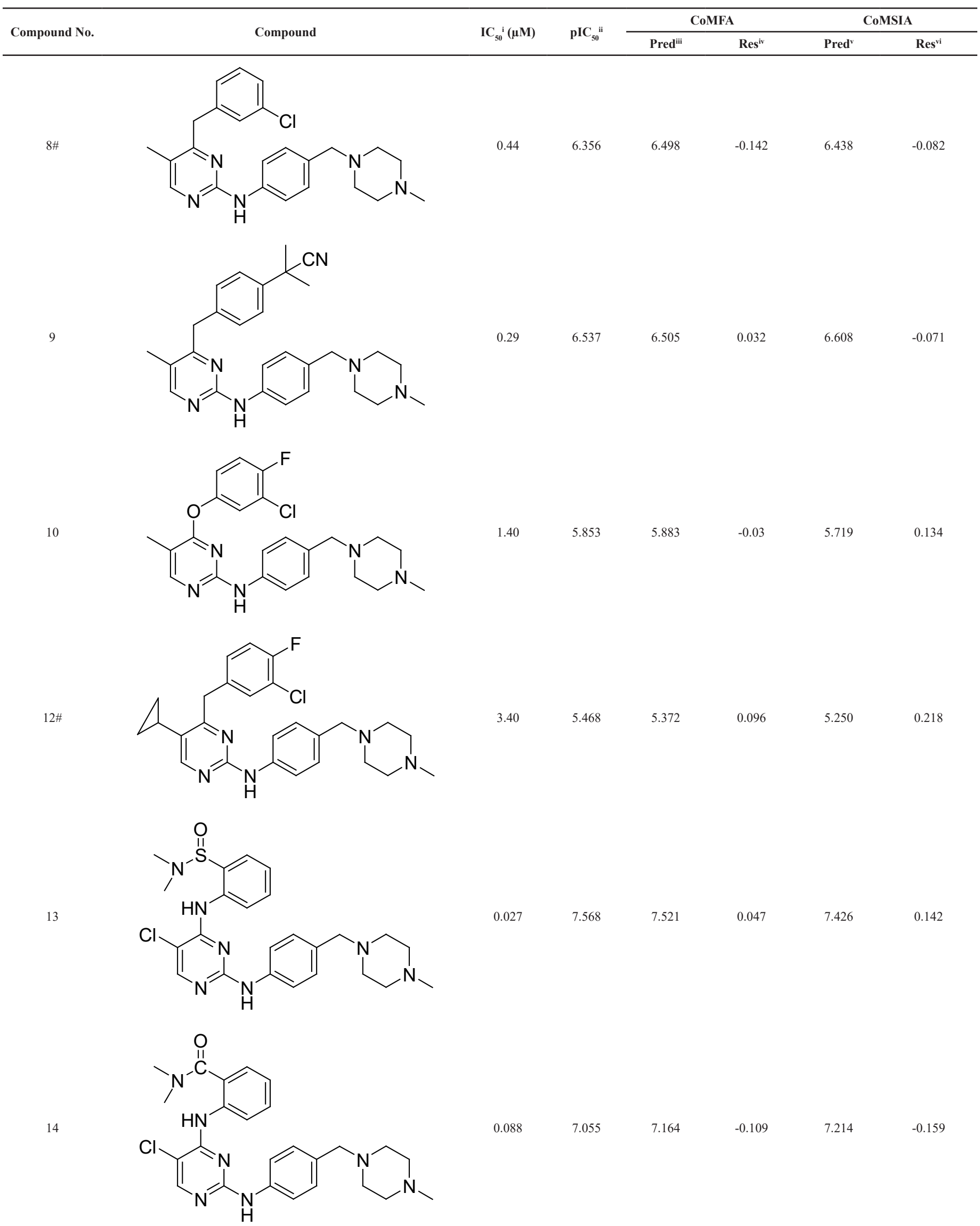




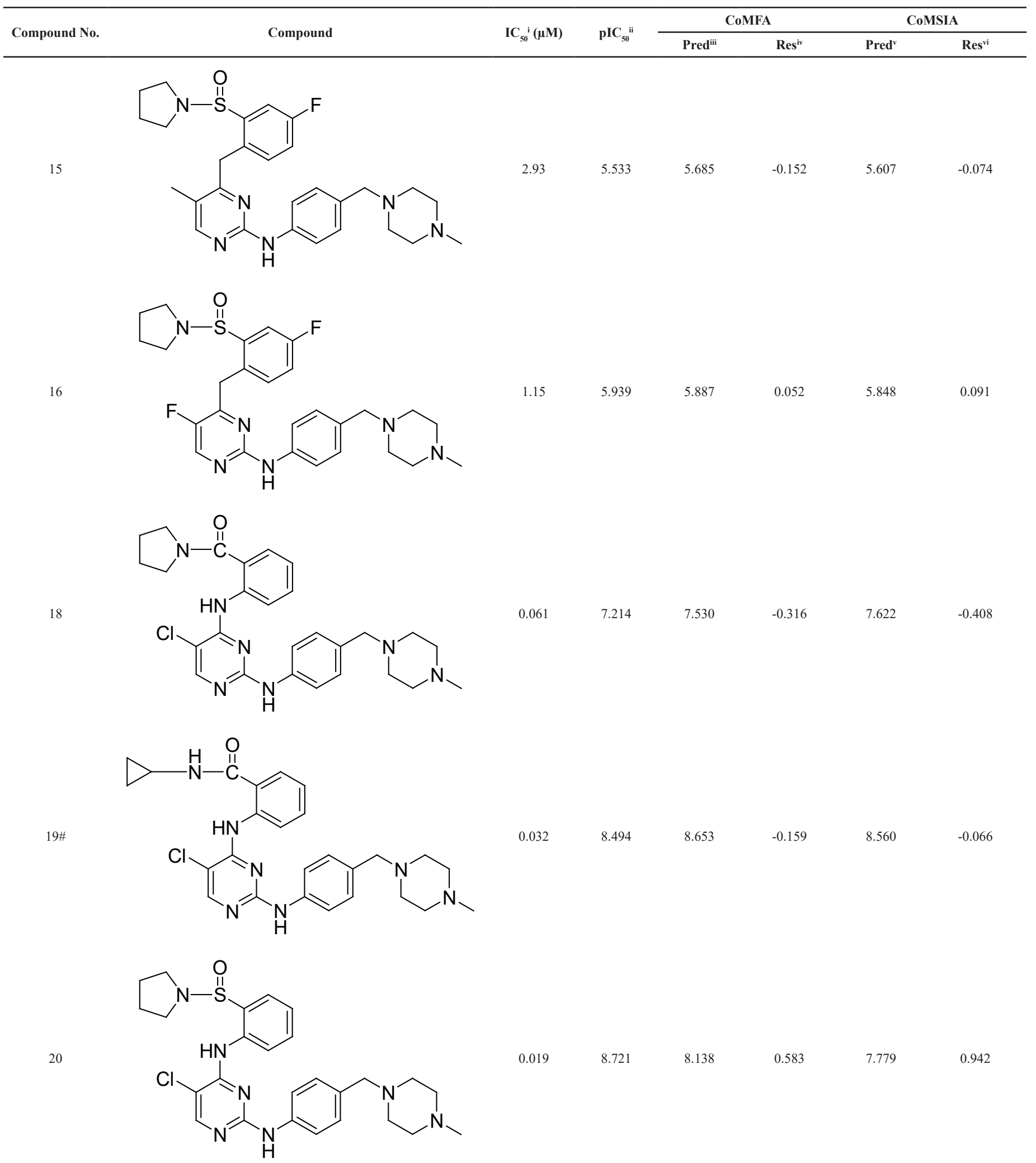




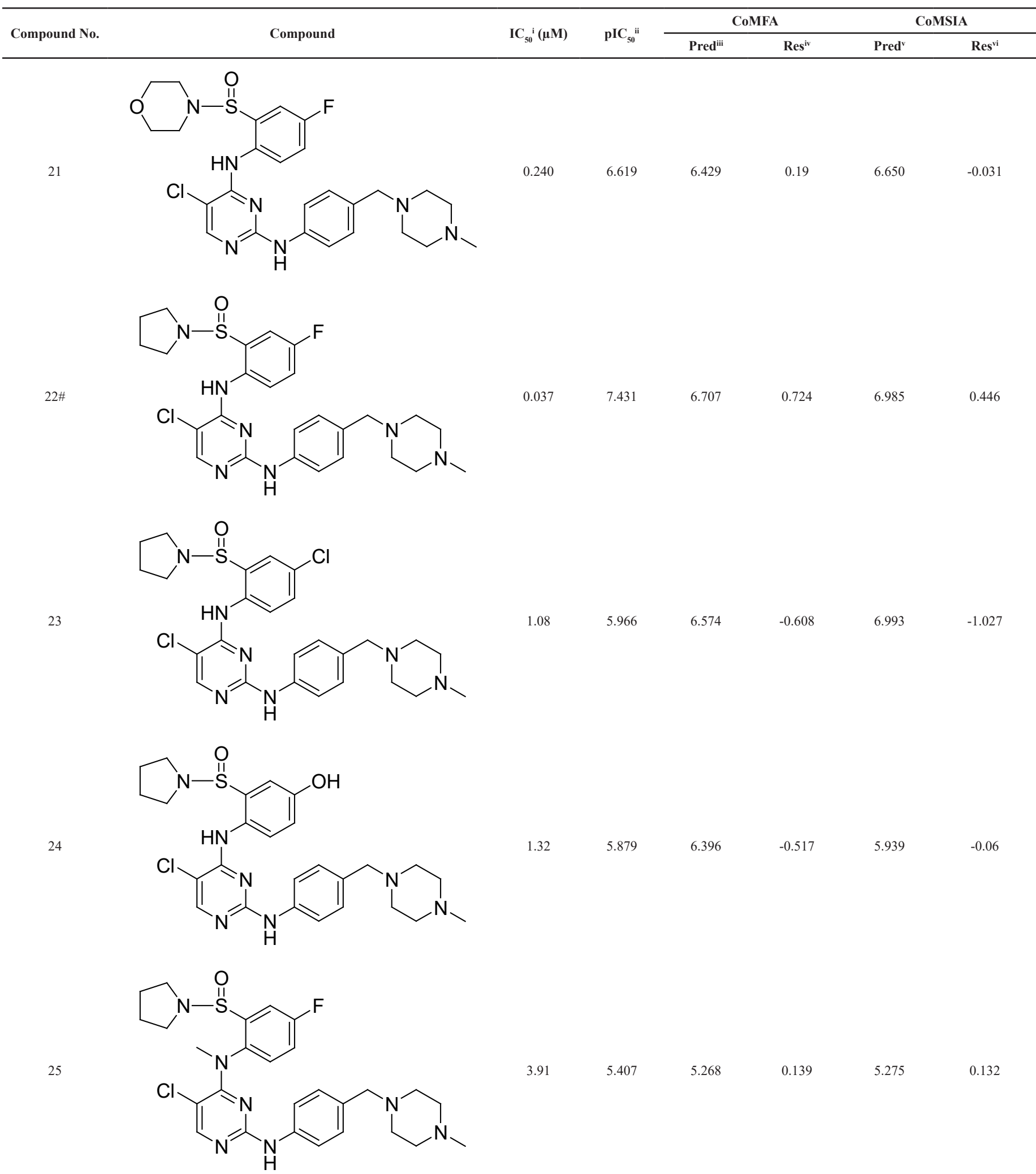




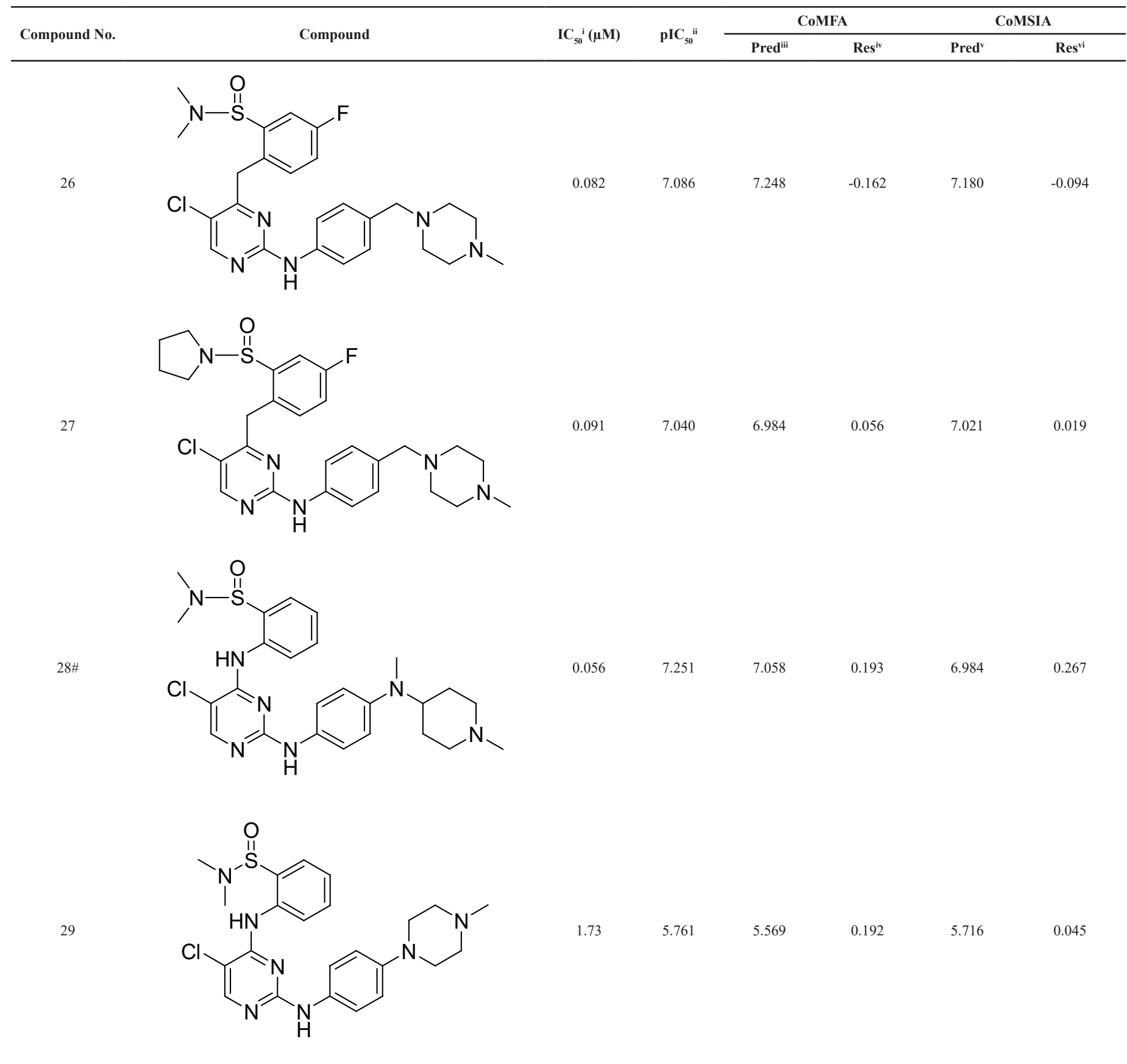

${ }^{\#}$ Test set compounds, ${ }^{\mathrm{i}} \mathrm{AXL}$ kinase inhibitory activity $\mathrm{IC}_{50}(\mu \mathrm{M})$, , ${ }^{\mathrm{ij}} \mathrm{pC} \mathrm{C}_{50}$ values $\left(-\log \mathrm{IC}_{50}\right)$, iii Predicted values based on CoMFA analysis, ${ }^{\text {iv }}$ Residual values based on

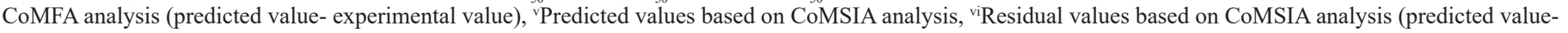
experimental value).

\section{RESULTS AND DISCUSSION}

\section{Results of CoMFA model}

CoMFA model was developed using steric and electrostatic fields. As the first step, the partial least square analysis was undertaken using "leave-one-out (LOO)". The value of $\mathrm{q}^{2}$ was found to be 0.700 with 5 optimum numbers of components. The same 5 components along with column filtering set to 2.0 were used to calculate the value of the conventional correlation coefficient $\left(\mathrm{r}^{2}{ }_{\text {ncv }}\right)$. The value of $r^{2}$ ncv was found to be 0.911; the value of $F$ was found to be 38.856; the value of SEE (Standard Error of Estimate) was found to be 0.316 . The steric and electrostatic field values were found to be 2.605 and 3.030 respectively. Table 2 depicts the statistical parameters obtained by development of the CoMFA model. The results of cross-validation coefficient $r^{2}{ }_{c v}(0.641)$ and bootstrap analysis $r_{b s}^{2}(0.957)$ supported the reliability of the developed QSAR model. Experimental and predicted activities of training set compounds and test set compounds are already represented in Table 1. Figure 4 (A, B) depicts the correlation of experimental and predicted activities of training and test set compounds. 

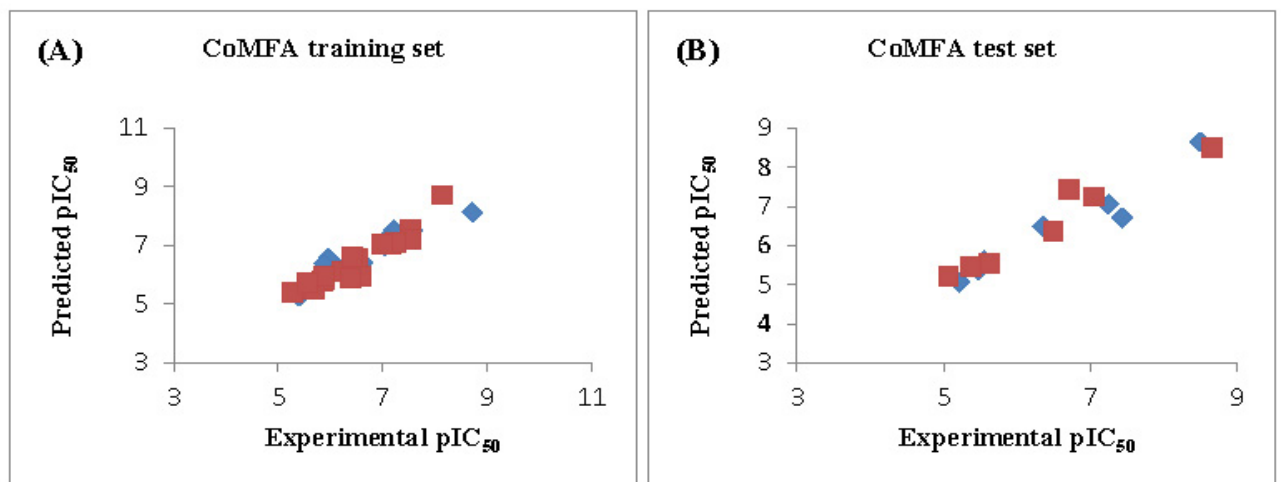

Fig. 4: Plot of experimental and predicted activities of the training and the test set compounds based on CoMFA model.
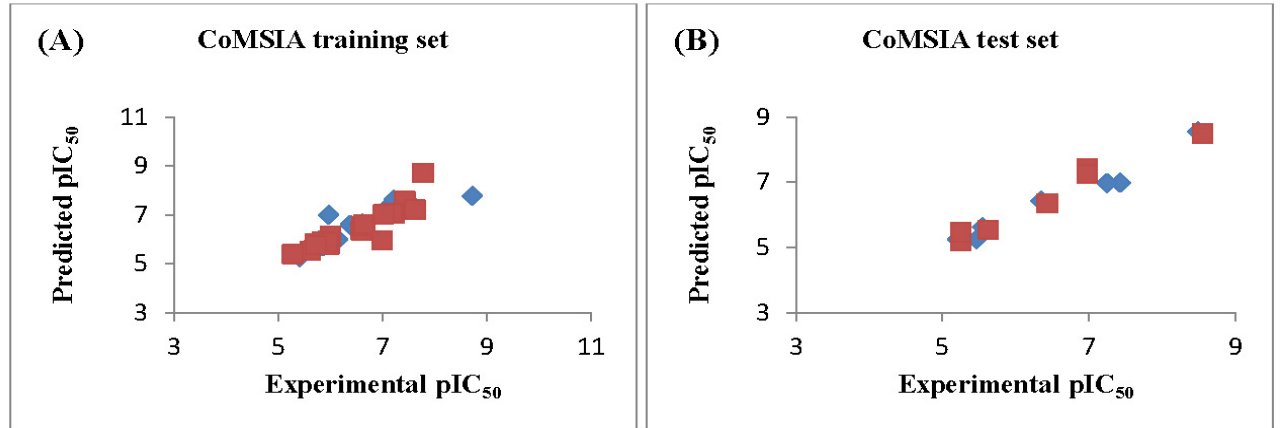

Fig. 5: Plot of experimental and predicted activities of the training and the test set compounds based on CoMSIA model.

\section{Results of CoMSIA model}

CoMSIA provides information of steric, electrostatic, hydrophobic, H-bond donor and H-bond acceptor fields. Comparative molecular field similarity indices analysis was undertaken by similar training and test set compounds as that of CoMFA following the successful results of CoMFA. The value of $\mathrm{q}^{2}$ was found to be 0.622 with 6 optimum numbers of components. To calculate the conventional correlation coefficient, same 6 components and column filtering as 2.0 were used. The value of $\mathrm{r}^{2}$ ncv was found to be 0.875 . The contribution of steric, electrostatic, hydrophobic, H-bond donor and $\mathrm{H}$-bond acceptor were found to be $0.666,1.571,0.985,1.015$ and 0.884 respectively. Further, bootstrap analysis $\left(\mathrm{r}_{\mathrm{bs}}^{2}=0.948\right)$ supported the quality of the developed CoMSIA model. To check internal reliability within the dataset, cross-validation by two groups (leave half out method) was undertaken. The $\mathrm{r}^{2}$ was found to be 0.595 . The values of $\mathrm{r}^{2}$ bs and $\mathrm{SEE}_{\mathrm{bs}}$ were found to be 0.948 and 0.256 respectively. Statistical parameters based on CoMFA and CoMSIA model were depicted in Table 2. Experimental and predicted activities of training and test set compounds are represented in Table 1. Figure $5(\mathrm{~A}, \mathrm{~B})$ depicts the correlation of experimental and predicted activities of the training and the test set compounds.

\section{QSAR visualization}

$3 \mathrm{D}$ contour maps are the important features of the comparative molecular field analysis (CoMFA) and comparative molecular similarity indices analysis (CoMSIA). These contour maps are derived when there are changes in molecular fields. The 3D space contour maps surrounding the compounds were derived for CoMFA as well as CoMSIA model. Based on the contour maps study, changes can be carried out to improve the biological activity and to optimize pyrimidine derivatives as AXL kinase inhibitors. Most active compound 20 was bifurcated into three equal parts to understand the effect of various fields on biological activity, (Figure 6A-C).

Table 2: Statistical parameters based on CoMFA and CoMSIA model.

\begin{tabular}{ccc}
\hline PLS analysis parameters & CoMFA & CoMSIA \\
\hline $\mathrm{r}_{\text {LOO }}{ }^{2}\left(\mathrm{q}^{2}\right)$ & 0.700 & 0.622 \\
$\mathrm{r}^{2}{ }_{\text {ncv }}$ & 0.911 & 0.875 \\
$\mathrm{SEE}$ & 0.316 & 0.384 \\
ONC & 5 & 6 \\
F value & 38.865 & 21.067 \\
Steric field contribution & 2.605 & 0.066 \\
Electrostatic field contribution & 3.030 & 1.571 \\
Hydrophobic field contribution & - & 0.985 \\
H-bond donor field contribution & - & 1.015 \\
H-bond acceptor field contribution & - & 0.884 \\
$\mathrm{r}^{2}{ }_{\mathrm{bs}}$ & 0.957 & 0.948 \\
$\mathrm{SEE}_{\mathrm{bs}}$ & 0.200 & 0.256 \\
$\mathrm{r}^{2}{ }_{\mathrm{CV}}$ & 0.641 & 0.595 \\
Test set ${ }^{2}\left(\mathrm{r}^{2}{ }_{\text {pred }}\right)$ & 0.709 & 0.668 \\
\hline
\end{tabular}




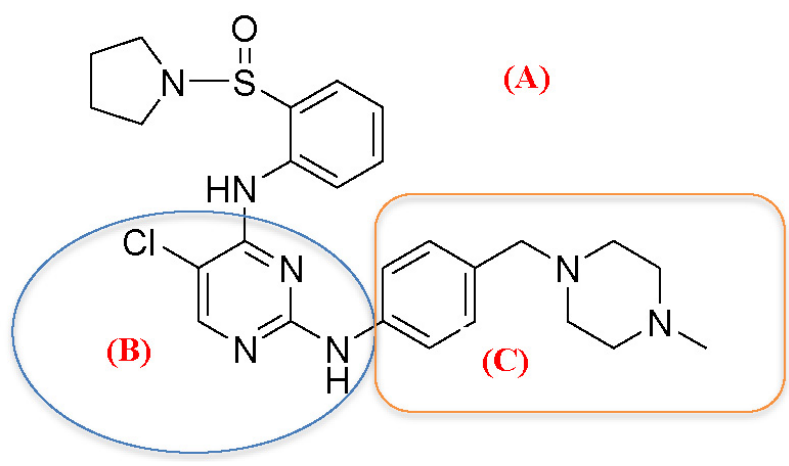

Fig. 6: Compound 20 bifurcated into three parts (A), (B) and (C).
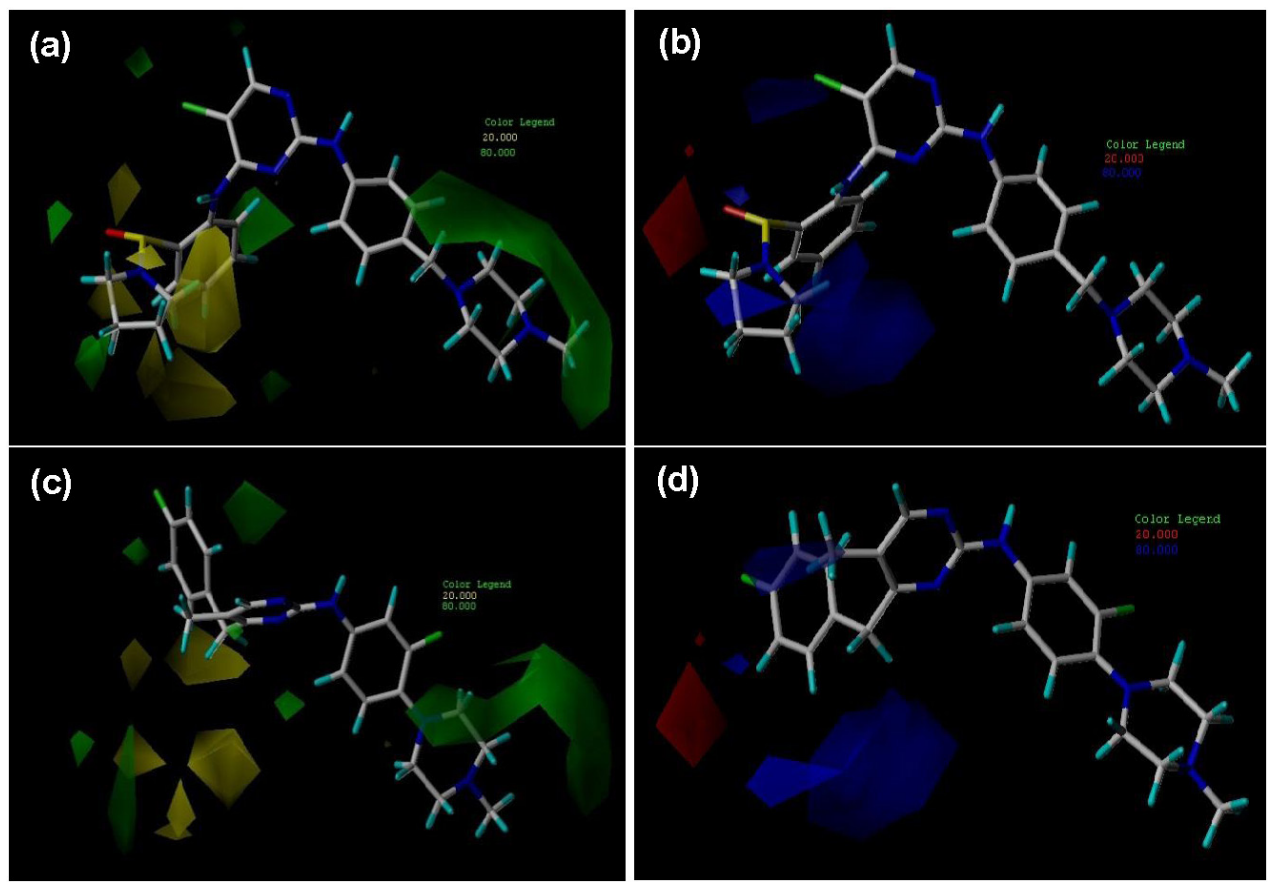

Fig. 7: CoMFA (StDev*Coeff) Contour maps of steric field ( $a, c)$ and electrostatic fields $(b, d)$ for compound $20(a, b)$ and the compound 4 (c, d) respectively.

\section{CoMFA contour maps}

Most active compound $20\left(\mathrm{IC}_{50}=0.019 \mu \mathrm{M}\right)$ was selected from the series for the examination of CoMFA contour maps. Contour maps were generated surrounding the molecule. Here, style of contour map was set as transparent for visualization of contours nearby the molecule. Green and yellow contour maps ( $80 \%$ and $20 \%$ contributions) represent the steric field. The green area indicates the favorable steric groups, and the inhibitory activity can be improved against AXL kinase receptor by incorporating the bulky groups. While, a yellow contour indicates the non-favorable steric groups for the activity, so the presence of bulky groups near this area can reduce biological activity. The electrostatic field with blue contours depicts positively charged groups. The electrostatic field with red contours depicts negatively charged groups.

Green contour near "C" region, in Figure 7(a), at piperazine ring, suggests that the bulky groups are favorable for the activity. Therefore, N-substituted piperazine ring may increase the biological activity. Green contour near " $\mathrm{C}$ " region at the $\mathrm{C}-4$ place of phenyl ring indicates that bulky groups are favored for inhibitory effect. This is apparent from actual biological activity values of compound $3\left(\mathrm{IC}_{50}=2.80 \mu \mathrm{M}\right), 4$ $\left(\mathrm{IC}_{50}=6.10 \mu \mathrm{M}\right)$ and $29\left(\mathrm{IC}_{50}=1.73 \mu \mathrm{M}\right)$. These compounds have 1-methylpiperazine ring directly attached to $\mathrm{C}-4$ atom of phenyl ring as compared to other compounds such as $8\left(\mathrm{IC}_{50}=0.44 \mu \mathrm{M}\right)$, $14\left(\mathrm{IC}_{50}=2.80 \mu \mathrm{M}\right), 18\left(\mathrm{IC}_{50}=0.061 \mu \mathrm{M}\right), 19\left(\mathrm{IC}_{50}=0.032 \mu \mathrm{M}\right.$, and $28\left(\mathrm{IC}_{50}=0.056 \mu \mathrm{M}\right)$ in which 1,4-dimethylpiperazine ring was present. This leads to the difference in inhibitory activity values of compounds. This is also evident from the comparison of compound 28's $\left(\mathrm{IC}_{50}=0.056 \mu \mathrm{M}\right)$ and 29's $\left(\mathrm{IC}_{50}=1.73 \mu \mathrm{M}\right)$ activities. Additionally, green contour at phenyl ring (C-5) in "A" region indicates that the bulky groups at this position results in increased inhibition. As seen in Figure 7(a), yellow contour are seen at the C-4 position in phenyl ring at " $\mathrm{A}$ " region, which suggests that incorporation of bulky groups are not favored. The bulky groups at C-5 position indicate increased inhibitory effect. This is apparent from the actual biological activity values of compounds $13,14,18,19,20,28$ in which no substitution was present at the $\mathrm{C}-5$ position so, they have very potent activity, 
while those compound with $-\mathrm{F}$ as substituent has minimal activity.

The electrostatic field represents blue and red contour maps. Blue contour favors positively charged groups and red contour map favors negatively charged groups. Most potent compound 20 was chosen as reference compound from the series.
The blue contour around phenyl ring at C-4 and C-5 position in "A" region suggests that addition of positively charged substituent results in an increase in the biological effect. (Figure 7(b)) This is apparent from relatively more active un-substituted phenyl ring in compounds 13,14,18,19, 20 as compared with the presence of negatively charged substituent in compounds $15,16,21$, and 25.
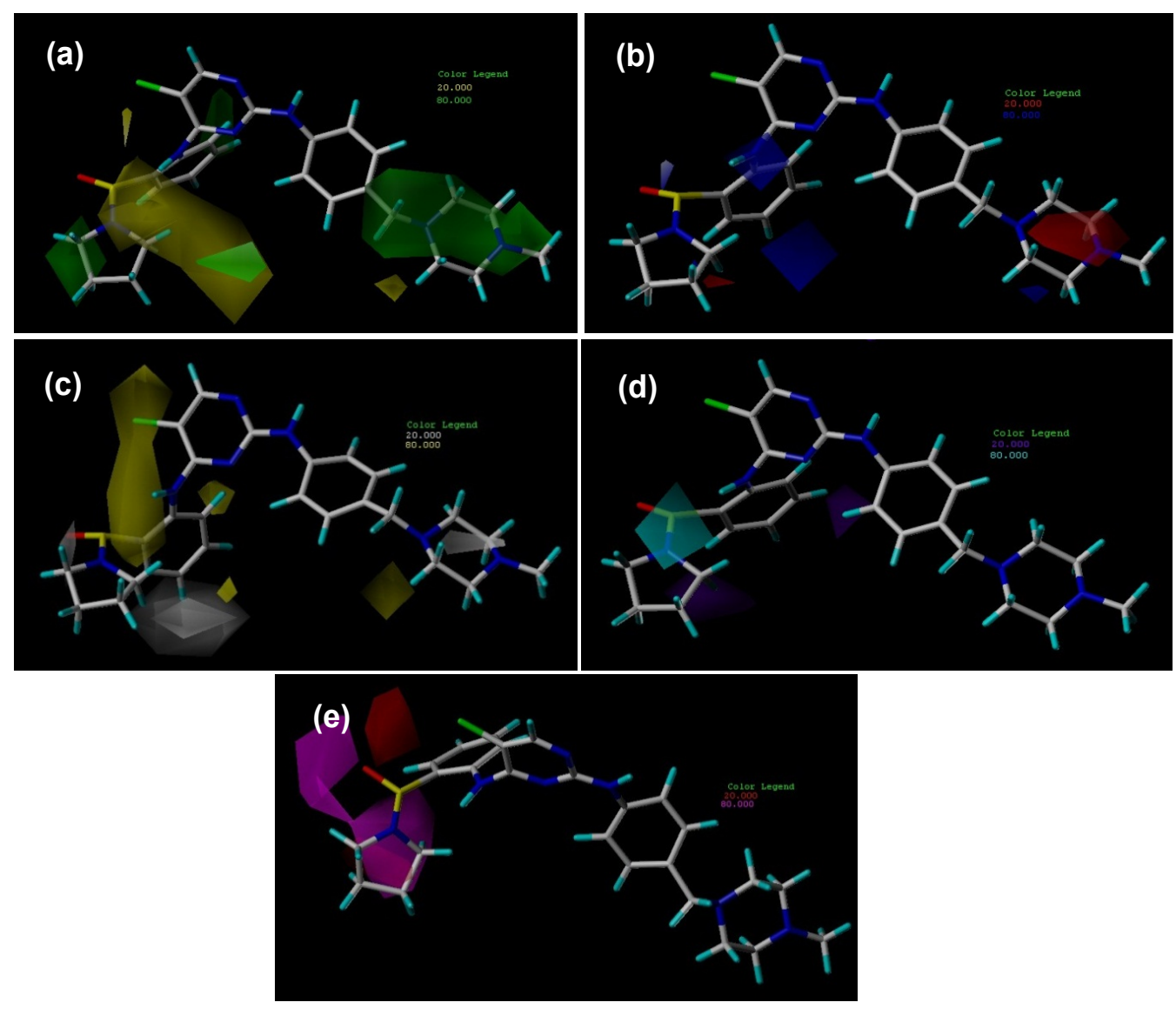

Fig. 8: CoMSIA (stDev*Coeff) contour maps of (a) steric, (b) Electrostatic (c) Hydrophobic (d) Hydrogen bond donor and (e) Hydrogen bond acceptor fields for compound 20 .

\section{CoMSIA contour maps}

Steric field and electrostatic field contour maps observed in the comparative molecular field similarity indices analysis (CoMSIA) are homogenous to those observed in CoMFA. In CoMSIA steric maps, the yellow contour at the $\mathrm{C}-2$ position in phenyl ring and at pyrrolidine ring in " $\mathrm{A}$ " region suggests that incorporation of bulky groups are not favored. This is apparent from the comparison of the biological effect of moderately active compound $21\left(\mathrm{IC}_{50}=0.240 \mu \mathrm{M}\right)$ and compound $26\left(\mathrm{IC}_{50}=0.082\right.$ $\mu \mathrm{M})$.

As it appears in Figure 8(b), red contour near piperazine ring in the $\mathrm{C}$ region, suggests that substituent with negative charge leads to enhancement of the inhibitory effect of compounds. So, an introduction of $-\mathrm{F},-\mathrm{Cl}$ or $-\mathrm{CF}_{3}$ groups may lead to a significant change in the inhibitory activity of compounds.

In Figure 8(c), white contour at the C-4 of phenyl scaffold in "A" region suggests that the hydrophilic substitution in this region results in increased biological activity. This is clear from the contour maps of the molecule, where bulky substitution such as $-\mathrm{F}$ and $-\mathrm{Cl}$ are present, leading to a reduction in activity. Further, yellow contours near the pyrimidine ring indicates the hydrophobic substitution, which leads to an increase in activity.

Cyan and purple contour maps represent the CoMSIA donor fields. H-bond donor substituents are favored in the region where the cyan contour map is present. While purple contour suggests that H-bond donor reduces the biological activity. Small cyan contour map in Figure 8(d), was observed at the C-2 position in phenyl ring which suggests that the presence of $\mathrm{H}$-bond donor in this region results in increased biological activity. Acceptor field represents magenta and red contour maps. H-bond acceptor substituents are favored in a region where the magenta contour is present. While H-bond acceptor substituents are not favored where the red contour is present. In Figure 8(e), the pyrrolidine ring in "A" region contains magenta contour map which suggests that the H-bond acceptor are favored for the activity. Important finding of QSAR study are depicted in Figure 9. 


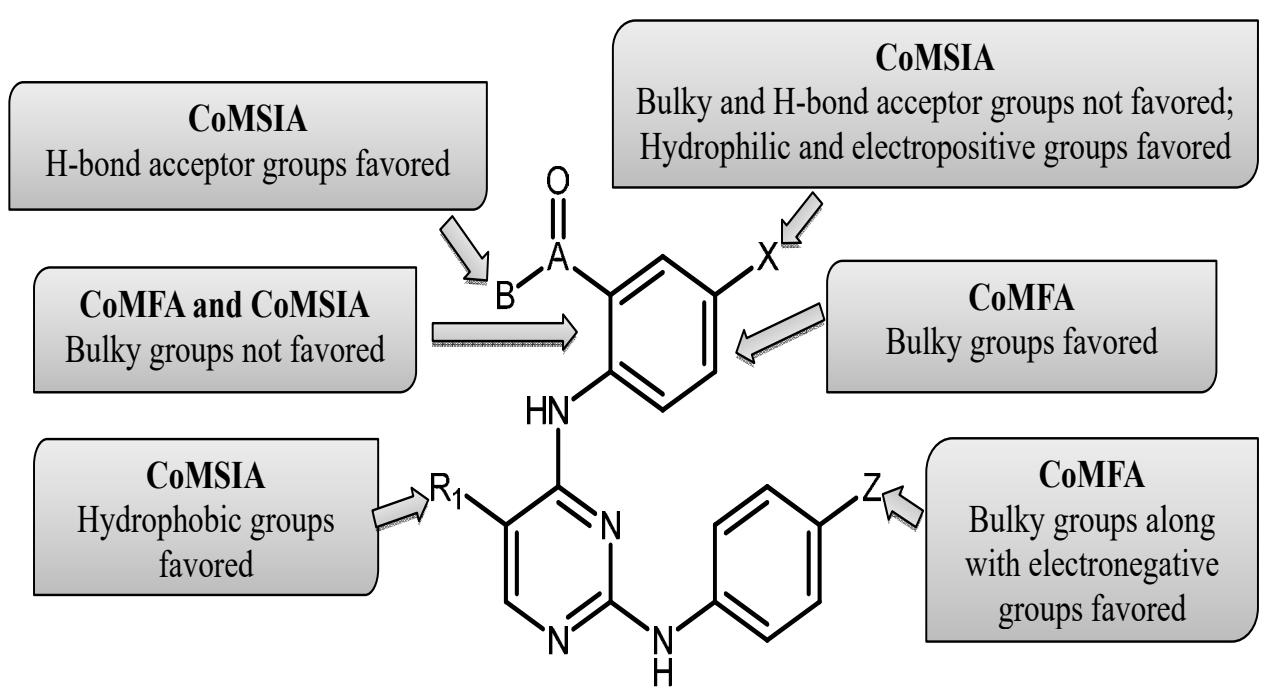

Fig. 9: Important structure finding and SAR from QSAR study.

\section{CONCLUSION}

Currently, AXL tyrosine kinase receptor is an emerging target for anti-cancer drug development. We undertook the quantitative structure-activity relationship analysis using CoMFA and CoMSIA to build the correlation between pyrimidine derivatives and their biological activity as AXL kinase inhibitors. To achieve CoMFA and CoMSIA, PLS analysis was undertaken to correlate the descriptor with biological activity. The crossvalidation coefficient $\left(\mathrm{q}^{2}\right)$ value of 0.700 and 0.622 for CoMFA and CoMSIA model along with all the statistical parameters and generated contour maps strongly reveal that the developed models are satisfactory. Contour maps indicate the important structural features such as incorporation of bulky groups along with negatively charged substituents at " $C$ " region in phenyl ring and the positively charged substituent at " $\mathrm{A}$ " region. These features lead to the significant enhancement of the biological activity. CoMFA and CoMSIA analysis strongly suggest that steric, electrostatic and hydrophobic fields play a crucial role in biological activity. 3D-QSAR analysis discussed in this study can be used further for the discovery of novel pyrimidine analogs as AXL kinase inhibitors as anti-cancer agents with a high degree of selectivity and specificity.

\section{ABBREVIATIONS}

$\begin{array}{ll}\text { RTK } & \text { Receptor Tyrosine Kinase } \\ \text { QSAR } & \text { Quantitative Structure Activity Relationships } \\ \text { NSCLC } & \text { Non-Small Cell Lung Cancer } \\ \text { CoMFA } & \text { Comparative Molecular Field Analysis } \\ \text { CoMSIA } & \text { Comparative Molecular Similarity Indices Analysis } \\ \text { PLS } & \text { Partial Least Square Analysis } \\ \text { LOO } & \text { Leave One Out } \\ \text { ONC } & \text { Optimum Number of Components } \\ \text { GH } & \text { Gasteiger Huckel } \\ r^{2}{ }_{n c v} & \text { Non-cross validation correlation coefficient }\end{array}$

$\begin{array}{ll}\mathrm{r}_{\text {pred }}^{2} & \text { Predictive correlation coefficient } \\ \mathrm{q}^{2} & \text { Cross-validation correlation coefficient } \\ \text { SEE } & \text { Standard Error of Estimate } \\ \text { F value } & \text { Fisher's value }\end{array}$

\section{ACKNOWLEDGMENTS}

The authors are grateful to Dr. S. S. Kadam, Chancellor, Bharati Vidyapeeth (Deemed to be University), Pune and Dr. K. R. Mahadik, Principal, Poona College of Pharmacy, Pune for their encouragement.

\section{AUTHOR CONTRIBUTIONS}

Both the authors, Siddharth J. Modi and Vithal M. Kulkarni, have approved the final version of the manuscript.

\section{CONFLICT OF INTEREST}

The authors declare no conflict of interest pertaining to this manuscript.

\section{REFERENCES}

Ben-Batalla I, Schultze A, Wroblewski M, Erdmann R, Heuser $\mathrm{M}$, Waizenegger JS, et al. Axl, a prognostic and therapeutic target in acute myeloid leukemia mediates paracrine crosstalk of leukemia cells with bone marrow stroma. Blood, 2013; 122(13):2443-52.

Buolamwini JK, Assefa H. CoMFA and CoMSIA 3D QSAR and docking studies on conformationally-restrained cinnamoyl HIV-1 integrase inhibitors: exploration of a binding mode at the active site. Journal of medicinal chemistry, 2002; 45(4):841-52.

Bush BL, Nachbar RB. Sample-distance partial least squares: PLS optimized for many variables, with application to CoMFA. Journal of computer-aided molecular design, 1993; 7(5):587-619.

Clark M, Cramer RD, Van Opdenbosch N. Validation of the general purpose Tripos 5.2 force field. Journal of Computational Chemistry, 1989; 10(8):982-1012.

Cramer RD, Patterson DE, Bunce JD. Comparative molecular field analysis (CoMFA). 1. Effect of shape on binding of steroids to carrier proteins. Journal of the American Chemical Society, 1988; 110(18):595967. 
Eder JP, Shapiro GI, Appleman LJ, Zhu AX, Miles D, Keer H, et al. A phase I study of foretinib, a multi-targeted inhibitor of c-Met and vascular endothelial growth factor receptor 2. Clinical Cancer Research, 2010; 16(13):3507-16.

Gay CM, Balaji K, Byers LA. Giving AXL the axe: targeting AXL in human malignancy. British journal of cancer, 2017; 116(4):415.

Golbraikh A, Tropsha A. Beware of q2!. Journal of molecular graphics and modelling, 2002; 20(4):269-76.

Graham DK, DeRyckere D, Davies KD, Earp HS. The TAM family: phosphatidylserine-sensing receptor tyrosine kinases gone awry in cancer. Nature Reviews Cancer, 2014; 14(12):769.

Hart CD, De Boer RH. Profile of cabozantinib and its potential in the treatment of advanced medullary thyroid cancer. OncoTargets and therapy, 2013; 6:1.

Hong CC, Lay JD, Huang JS, Cheng AL, Tang JL, Lin MT, et al. Receptor tyrosine kinase AXL is induced by chemotherapy drugs and overexpression of AXL confers drug resistance in acute myeloid leukemia. Cancer letters, 2008; 268(2):314-24.

Klebe G, Abraham U, Mietzner T. Molecular similarity indices in a comparative analysis (CoMSIA) of drug molecules to correlate and predict their biological activity. Journal of medicinal chemistry, 1994; 37(24):4130-46.

Lai C, Gore M, Lemke G. Structure, expression, and activity of Tyro 3, a neural adhesion-related receptor tyrosine kinase. Oncogene, 1994; 9:2567-78.

Lee HJ, Jeng YM, Chen YL, Chung L, Yuan RH. Gas6/Axl pathway promotes tumor invasion through the transcriptional activation of Slug in hepatocellular carcinoma. Carcinogenesis, 2013; 35(4):769-75.

Liu J, Zhang H, Xiao Z, Wang F, Wang X, Wang Y. Combined 3D-QSAR, molecular docking and molecular dynamics study on derivatives of peptide epoxyketone and tyropeptin-boronic acid as inhibitors against the $\beta 5$ subunit of human $20 \mathrm{~S}$ proteasome. International journal of molecular sciences, 2011; 12:1807-35.

Lu P, Wei X, Zhang R. CoMFA and CoMSIA 3D-QSAR studies on quionolone caroxylic acid derivatives inhibitors of HIV-1 integrase. European journal of medicinal chemistry, 2010; 45(8):3413-9.

Mahadevan D, Cooke L, Riley C, Swart R, Simons B, Della Croce $\mathrm{K}$, et al. A novel tyrosine kinase switch is a mechanism of imatinib resistance in gastrointestinal stromal tumors. Oncogene, 2007; 26(27):3909.

Makhija MT, Kulkarni VM. 3D-QSAR and molecular modeling of HIV-1 integrase inhibitors. Journal of computer-aided molecular design, 2002; 16(3):181-200

Mollard A, Warner SL, Call LT, Wade ML, Bearss JJ, Verma A, et al. Design, synthesis, and biological evaluation of a series of novel AXL kinase inhibitors. ACS medicinal chemistry letters, 2011; 2(12):907-12.

Mori M, Kaneko N, Ueno Y, Tanaka R, Cho K, Saito R, et al. ASP2215, a novel FLT3/AXL inhibitor: Preclinical evaluation in acute myeloid leukemia (AML). Clin Oncol, 2014; 32:7070.

Myint KZ, Xie XQ. Recent advances in fragment-based QSAR and multi-dimensional QSAR methods. International journal of molecular sciences, 2010; 11(10):3846-66.

O'Bryan JP, Frye RA, Cogswell PC, Neubauer A, Kitch B, Prokop $\mathrm{C}$, et al. Axl, a transforming gene isolated from primary human myeloid leukemia cells, encodes a novel receptor tyrosine kinase. Molecular and cellular biology, 1991; 11(10):5016-31.

Ou WB, Corson JM, Flynn DL, Lu WP, Wise SC, Bueno R, et al. AXL regulates mesothelioma proliferation and invasiveness. Oncogene, 2011; 30(14):1643.

Paccez JD, Vasques GJ, Correa RG, Vasconcellos JF, Duncan $\mathrm{K}, \mathrm{Gu} \mathrm{X}$, et al. The receptor tyrosine kinase Axl is an essential regulator of prostate cancer proliferation and tumor growth and represents a new therapeutic target. Oncogene, 2013; 32(6):689.

Raichurkar AV, Kulkarni VM. Understanding the antitumor activity of novel hydroxysemicarbazide derivatives as ribonucleotide reductase inhibitors using CoMFA and CoMSIA. Journal of medicinal chemistry, 2003; 46(21):4419-27.

Sridhar J, Foroozesh M, Stevens CK. QSAR models of cytochrome P450 enzyme 1A2 inhibitors using CoMFA, CoMSIA and HQSAR. SAR and QSAR in Environmental Research, 2011; 22(7-8):68197.

SYBYL X Molecular Modeling Software, Tripos Associates, V. 2.0, St. Louis, USA, 2012; software available at http://www.tripos.com.

Yan SB, Peek VL, Ajamie R, Buchanan SG, Graff JR, Heidler SA, et al. LY2801653 is an orally bioavailable multi-kinase inhibitor with potent activity against MET, MST1R, and other oncoproteins, and displays anti-tumor activities in mouse xenograft models. Investigational new drugs, 2013; 31(4):833-44.

Yuen HF, McCrudden CM, Huang YH, Tham JM, Zhang X, Zeng Q, et al. TAZ expression as a prognostic indicator in colorectal cancer. PloS one, 2013; 8(1):e54211.

Zhang Y, Tang YJ, Man Y, Pan F, Li ZH, Jia LS. Knockdown of AXL receptor tyrosine kinase in osteosarcoma cells leads to decreased proliferation and increased apoptosis. International journal of immunopathology and pharmacology, 2013; 26(1):179-88.

Zhang Z, Lee JC, Lin L, Olivas V, Au V, LaFramboise T, et al. Activation of the AXL kinase causes resistance to EGFR-targeted therapy in lung cancer. Nature genetics, 2012; 44(8):852.

Zhou L, Liu XD, Sun M, Zhang X, German P, Bai S, et al. Targeting MET and AXL overcomes resistance to sunitinib therapy in renal cell carcinoma. Oncogene, 2016; 35(21):2687.

How to cite this article:

Modi SJ, Kulkarni VM. 3D-QSAR analysis of pyrimidine derivatives as AXL kinase inhibitors as anticancer agents. J App Pharm Sci, 2018; 8(11): 015-027. 